\title{
Coral Assemblages in the Southeastern Arabian Gulf (Qatar and Abu Dhabi, UAE): Various Stages of Acropora Recovery a Decade after Recurrent Elevated Temperature Anomalies
}

\author{
Kristi A. Foster ${ }^{1 *}$, Greg Foster ${ }^{1}$, Suaad Al-Harthi ${ }^{2}$ \\ ${ }^{1}$ Nova Southeastern University Oceanographic Center, Dania Beach, USA \\ ${ }^{2}$ Environment Agency-Abu Dhabi, Abu Dhabi, United Arab Emirates \\ Email: *kfoster@nova.edu
}

Received February 5, 2013; revised May 3, 2013; accepted May 22, 2013

Copyright (C) 2013 Kristi A. Foster et al. This is an open access article distributed under the Creative Commons Attribution License, which permits unrestricted use, distribution, and reproduction in any medium, provided the original work is properly cited.

\begin{abstract}
This study describes the coral communities near Qatar and Abu Dhabi (UAE) ten years after the recurrent elevated temperature anomalies of 1996, 1998 and 2002 which resulted in the mass mortality of Acropora spp. Data derived from photo transects taken over a four-year period were analyzed to characterize the existing coral communities, to compare these to the pre- and post-disturbance communities in the adjacent waters near Dubai, and to project the timeframes required for the communities to return to pre-disturbance levels. The massive corals, dominated by Porites spp. and faviids, showed no long-terms affects associated with exposures to the three thermal anomalies; whereas acroporids, comprising $0 \%-8 \%$ of the live coral cover, were in various stages of recovery. Projections indicated that acroporid regeneration will require 15 - 32 years to achieve the $\geq 40 \%$ pre-disturbance area cover. The existing communities are too small in size and number to be self-seeding; thus, they are dependent upon the recruitment of larvae from remote refuges of colonies that survived the thermal anomalies. Efforts to identify these refuges and to establish appropriate multi-national conservation programs shall become critical to the future survival of acroporids throughout the southeastern Arabian Gulf as the projected regeneration times exceed the periods between disturbances, which are expected to occur more frequently as a result of global climate change.
\end{abstract}

Keywords: Coral Community; Elevated Temperature Anomaly; Mass Mortality; Acropora; Arabian Gulf; Recovery

\section{Introduction}

The 1998 elevated sea surface temperature anomaly was associated with widespread coral bleaching and mortality around the world (e.g. [1-6]). While the Indo-Pacific suffered heavy losses of coral cover, the Arabian Gulf (a semi-enclosed sea that is connected to the Indian Ocean via the Strait of Hormuz) experienced minimal losses, primarily because many thermally-susceptible corals had died during an elevated temperature anomaly (ETA) two years earlier in $1996[1,7,8]$. The subsequent 1998 ETA and a third anomaly that occurred in 2002 had only minor effects on the remaining coral communities, because the surviving colonies (primarily massive Porites, Favia, and Platygyra spp.) were relatively resistant to the elevated temperatures [8]. Arabian Gulf corals are regularly exposed to natural conditions that ${ }^{*}$ Corresponding author. exceed survival threshold limits of corals elsewhere in the world, with temperatures between $16.5^{\circ} \mathrm{C}-35.0^{\circ} \mathrm{C}$ and salinities $>40 \mathrm{ppt}$ (e.g. [9-11]). Exposure to temperature and salinity extremes has been selective for coral species that are adapted to such harsh conditions (i.e. less than one-third of the scleractinian species that are found in the neighboring Gulf of Oman have adapted to survive in the southeastern Arabian Gulf [7,12-14]). During the ETAs, temperatures in the Arabian Gulf were $>2^{\circ} \mathrm{C}$ above the typical summer maximum $[15,16]$ and $4^{\circ} \mathrm{C}-8^{\circ} \mathrm{C}$ above sea surface temperatures (SSTs) associated with coral bleaching elsewhere in the world [4-6, 17]. These increases in sea surface temperatures exceeded the thermal tolerances of the acroporid populations in the southeastern Arabian Gulf (i.e. near Dubai and Abu Dhabi) resulting in a loss of $>98 \%$ of the frame-building coral cover in some areas, the total mortality of seven Acropora species, and a shift in dominance from acro- 
porids to massive taxa $[7,8]$.

As ETAs are predicted to occur with greater frequency $[17,18]$, it is possible that future coral communities around the world may be in a constant state of recovery, with regeneration times exceeding the periods between disturbances. The coral assemblages within the southeastern Arabian Gulf, which have developed following three ETAs within a six-year period, are examples of such communities in which resistant massive taxa comprise the foundation of the hard coral habitat while the susceptible, yet resilient, acroporids fluctuate through various stages of recovery. The presence of Acropora juveniles within two years of the 1996 mass mortality indicated the potential for rapid recovery in the Arabian Gulf [8], but this initial recruitment was lost in some areas due to additional acroporid mortality during the 2002 ETA [19]. Despite this setback, Acropora had recovered sufficiently by 2006 for it to regain its status as the dominant taxa in several coral communities near Dubai (UAE) [19]. In other areas of the southeastern Arabian Gulf, such as near Abu Dhabi (UAE) and Qatar, Acropora populations are recovering at slower rates.

The objectives of this study are to 1) characterize the coral communities near Qatar and Abu Dhabi between 2006 and 2009; 2) compare these to the pre- and postdisturbance communities in the adjacent waters near Dubai [7,8,16,19,20]; and 3) determine whether projected regeneration times for acroporids exceed the frequency of disturbances in the southeastern Arabian Gulf.

\section{Methods}

\subsection{Monitoring Stations}

Monitoring site locations were selected in areas representing various shallow water coral communities within the southeastern Arabian Gulf including sites along the coasts, near offshore islands and on top of a limestone dome (Figure 1, Table 1). The stations consisted of three replicate $10.0 \mathrm{~m} \times 1.5 \mathrm{~m}$ belt transects. Each transect was permanently marked by stainless steel spikes cemented into the substrate. Temporary lines, taughtly strung between the markers, served as guidelines for photo transects. Annual transect photos were taken between October-November in 2006, 2008 and 2009 and between July-August in 2007. Logistics and weather conditions prevented certain sites from being monitored annually (Table 1).

$\mathrm{HOBO} \circledast$ temperature loggers, attached to a marker approximately $0.5 \mathrm{~m}$ above the benthos, recorded hourly seawater temperatures between September 2005 and November 2009. Loggers were deployed at thirteen sites with periodic retrieval and replacement. (Recorders were not deployed at Halul East or Hawksbill Reef.) The longest consecutive data collection occurred at Makaseb, from September 2005 through April 2009.

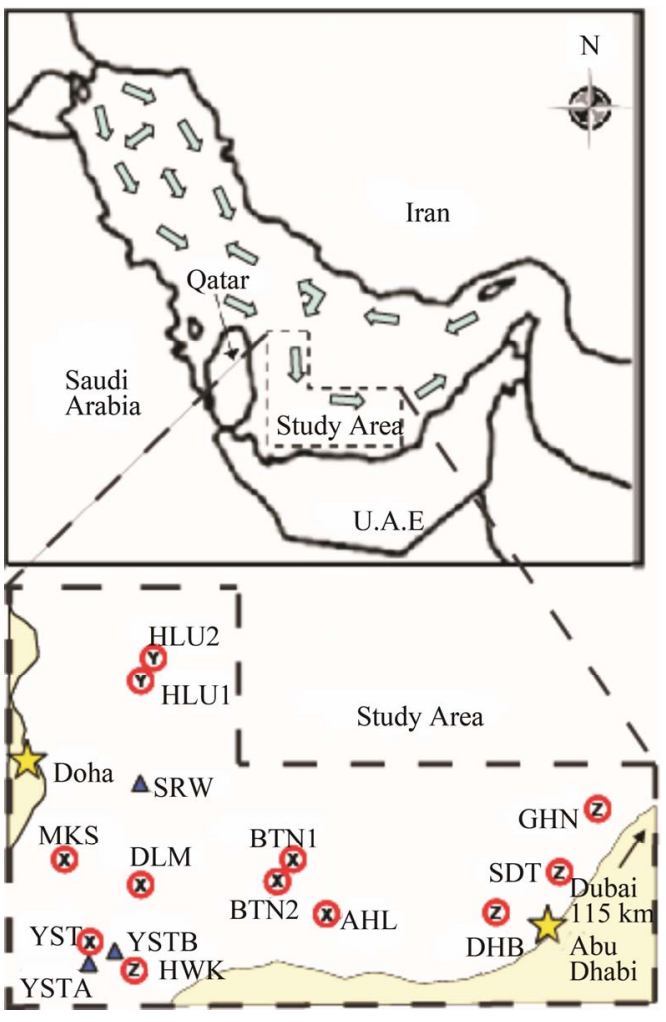

Figure 1. Map of monitoring stations in the southeastern Arabian Gulf; Map of Arabian Gulf and surrounding region with study area outlined. Arrows indicate prevailing surface currents, adapted from Reynolds (1993) [22]. Circles indicate monitoring station locations with coral assemblages $\mathrm{X}, \mathrm{Y}$ and $\mathrm{Z}$. Triangles indicate sites that had temperature recorders only.

\subsection{Image Analysis}

Digital images were taken along the belt transects using a rigid photo-framer $(0.5 \mathrm{~m} \times 0.75 \mathrm{~m}$ footprint $)$ that oriented the camera at normal incidence and at a fixed distance of $50 \mathrm{~cm}$ above the benthos. Forty images were joined into a single mosaic for each $10.0 \mathrm{~m} \times 1.5 \mathrm{~m}$ belt transect. Colonies were traced using Coral Point Count (CPCe) [21] and identified to genus. CPCe calculated colony surface areas (planar view) based on the known area of the benthic view within the photo-framer. Colony counts and surface areas for all transect images within a given year were pooled to provide coral densities and percent live coral cover. Massive colonies were grouped into five size-dependent classifications, based on surface areas, to provide size frequency distributions (Table 2). To determine the most appropriate groupings, size frequency distributions were compared for surface areas associated with increments of $r=1,2$, and $3 \mathrm{~cm}$. The size class frequencies based on increments of $\mathrm{r}=2 \mathrm{~cm}$ were normally distributed whereas those associated with $r=1$ $\mathrm{cm}$ and $\mathrm{r}=3 \mathrm{~cm}$ were skewed to the left and right, respectively. 
Table 1. Monitoring station descriptions.

\begin{tabular}{|c|c|c|c|c|c|}
\hline Station & Site Name & Depth (m) & Location & Data Collection & Year(s) \\
\hline HLU2 & Halul East & $4.8-5.2$ & Island & Single Assessment & 2006 \\
\hline HLU1 & Halul South & $4.5-5.3$ & Island & Single Assessment & 2006 \\
\hline FSH & Fasht Al-Ghabi & $3.5-3.9$ & Coastal & Single Assessment & 2006 \\
\hline SRW & Shra'aw & $5.0-5.5$ & Island & Temperature data & 2006 \\
\hline MKS & Makaseb & $2.1-5.5$ & Island & Repetitive Monitoring & 2006, 2009 \\
\hline YST & Yasat Ali & $3.0-4.7$ & Island & Repetitive Monitoring & 2006-09 \\
\hline YSTA & Yasat Asfl & $5.0-5.5$ & Island & Temperature data & 2006-07 \\
\hline YSTB & Yasat Buoy \#B-08 & 31 & Offshore & Temperature data & 2008 \\
\hline HWK & Hawksbill Reef & $7.0-8.5$ & Limestone Dome & Single Assessment & 2009 \\
\hline DELM5 & Delma & $5.3-5.6$ & Island & Single Assessment & 2009 \\
\hline BTN1 & Bu Tinah North & $1.8-3.6$ & Island & Repetitive Monitoring & 2006-09 \\
\hline BTN2 & Bu Tinah West & $2.0-3.5$ & Island & Repetitive Monitoring & 2006-08 \\
\hline AHL & Al Hiel & $2.6-4.2$ & Island & Repetitive Monitoring & 2006-09 \\
\hline DHB & Dhabiya & $6.4-7.2$ & Coastal & Single Assessment & 2009 \\
\hline SDT & Saadiyat & $5.7-7.2$ & Coastal & Repetitive Monitoring & 2007-09 \\
\hline GHN & Ras Ghanada & $7.6-8.5$ & Coastal & Repetitive Monitoring & 2007-09 \\
\hline
\end{tabular}

Station abbreviations coincide with those shown in Figure 1. Data collection regimes include single assessments, repetitive monitoring (annual data collection) and temperature data (no other benthic data collected) during the years specified.

Table 2. Size-dependent classifications for massive coral colonies.

\begin{tabular}{ccc}
\hline Size Class & Radius $(\mathrm{cm})$ & Surface Area $\left(\mathrm{cm}^{2}\right)$ \\
\hline 1 & $<2$ & $<12.7$ \\
2 & $2-4$ & $12.7-50.2$ \\
3 & $4-6$ & $50.3-113.0$ \\
4 & $6-8$ & $113.1-201.1$ \\
5 & $>8$ & $>201.1$ \\
\hline
\end{tabular}

Colonies are assumed to be circular $\left(\right.$ Area $\left.=\pi r^{2}\right)$.

\subsection{Coral Assemblages}

Post-disturbance coral assemblage distinctions followed the protocol used by Riegl (2002) in order to compare the results of this study to pre- and post-disturbance assemblages near Dubai $[8,19]$. Data processing for classification and ordination included 1) fourth root transformation for the production of a Bray-Curtis similarity matrix; 2) agglomerative, hierarchical cluster analysis using group average sorting; and 3) non-metric multi-dimensional scaling (nMDS). All multivariate analyses were implemented using PRIMER [23].

\subsection{Acropora Community Profile}

Repetitive monitoring provided surface areas for individual Acropora clathrata colonies between 2007 and 2009. A linear regression of the changes in colony surface area between time $(\mathrm{x})$ and time $(\mathrm{x}+1)$ produced a formula for annual Acropora growth rates during this disturbance-free period $\left(\mathrm{n}=13, \mathrm{r}^{2}=0.883\right)$ (Figure 2).

The linear regression-based growth rate formula and previously published growth rates of $5-10 \mathrm{~cm} \cdot \mathrm{yr}^{-1}$ for Acropora clathrata in the Arabian Gulf $[8,24]$ were used to project the recovery of the current Acropora assemblages to pre-disturbance levels. Projections are idealized forecasts that assume current parameters (e.g. environmental conditions, coral survival and growth rates) remain unchanged over time $[25,26]$. The recovery projecttions were based on the following conditions:

- "LR(7.5)" projections adhered to the linear regression formula until the growth rate reached $7.5 \mathrm{~cm} \cdot \mathrm{yr}^{-1}$ (i.e. the mid-point of previously reported annual Acropora growth rates), then stayed at $7.5 \mathrm{~cm} \cdot \mathrm{yr}^{-1}$ for the remainder of the projection period.

- All acroporids that were repetitively monitored in this study grew from year to year. This scenario is not likely to continue long-term as partial mortality is a 


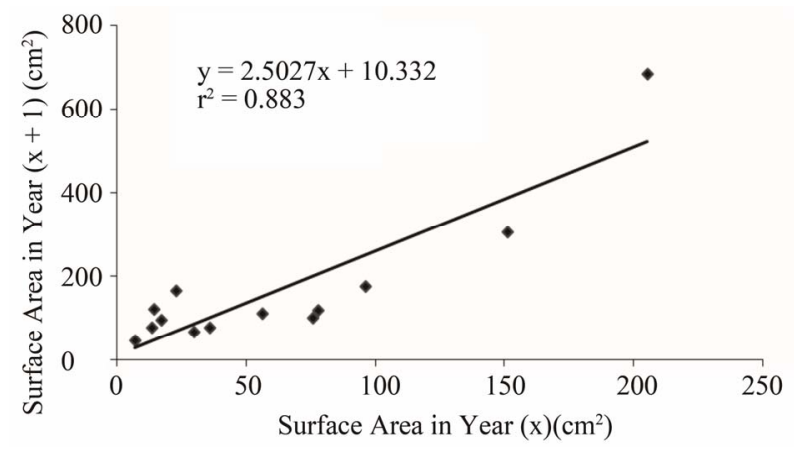

Figure 2. Growth rate for Acropora clathrata at Ras Ghanada (2007-2009); Solid line: linear regression to determine the surface area of an acroporid in year $(x+1)$ based on the surface area of the same individual the previous year.

normal component of coral population dynamics. Thus, an alternative scenario is presented whereby the partial mortality rates experienced by the massive corals within the same study area were applied to the acroporids (i.e. $40 \%$ of the acroporid colonies were chosen at random to lose $30 \%$ of their respective surface areas in a given year). Additional data are needed to establish the actual partial mortality rates for the acroporid populations under normal, nondisturbance conditions.

- Recruitment varied spatially and temporally, with six recruits being the maximum recorded (Ras Ghanada, 2009). Additional data are needed to determine the normal annual recruitment. Projections were made for zero, two and six recruits per year.

- The surface area of a new recruit was $47 \mathrm{~cm}^{2}$ (i.e. the mean surface area for 2008-2009 recruits measured in this study).

\section{Results}

\subsection{Environmental Setting}

Hourly temperature profiles from individual sites were pooled to provide mean temperature profiles for the region: 1) the normal annual temperature range was $16.5^{\circ} \mathrm{C}$ to $\left.35.0^{\circ} \mathrm{C}, 2\right)$ spring warming and autumn cooling rates were uniform across the region, ranging between $0.09^{\circ} \mathrm{C}-0.13^{\circ} \mathrm{C} /$ day and $0.10^{\circ} \mathrm{C}-0.15^{\circ} \mathrm{C} /$ day, respectively, and 3) the mean daily temperature range was $<2.5^{\circ} \mathrm{C}$. The prominent benthic taxa in these environments were hard corals, crustose coralline algae, encrusting sponges and oysters.

The coral communities did not experience mass mortalities during this study, indicating acclimatization to the lower and upper temperature limits described above. In July-August 2007, when water temperatures were $32^{\circ} \mathrm{C}$ $35^{\circ} \mathrm{C}$, a few individual coral colonies did exhibit signs of minor bleaching and yellow-band disease. (Yellow-band was only observed on Porites spp.) Subsequent visits in November 2007 revealed that 1) bleached corals had returned to normal coloration without mortality, and 2) disease resulted in mortality of affected polyps while the remainder of the colony survived.

\subsection{Coral Assemblages}

Cluster analysis differentiated three assemblages (designated $\mathrm{X}, \mathrm{Y}$ and $\mathrm{Z}$ ) of variable massive coral cover within the southeastern Arabian Gulf (Figures 3 and 4, Table 3).

$\mathrm{X}$ : This assemblage encompassed five sites near Abu Dhabi islands (Makaseb, Yasat, Bu Tinah, Al Hiel) that were sparsely populated with Porites harrisoni and other massive corals. Porites, Favia/Favites and Platygyra were the predominant genera. Subordinate genera included Cyphastrea, Leptastrea and Siderastrea. Live acroporids were not observed within these sites; however, consolidated rubble indicated that acroporids had existed at these sites at one time.

Y: This assemblage encompassed two sites near Halul Island, Qatar that were sparsely populated with massive species and tabular colonies of Acropora clathrata. Porites, Favia/Favites and Platygyra were the predo-
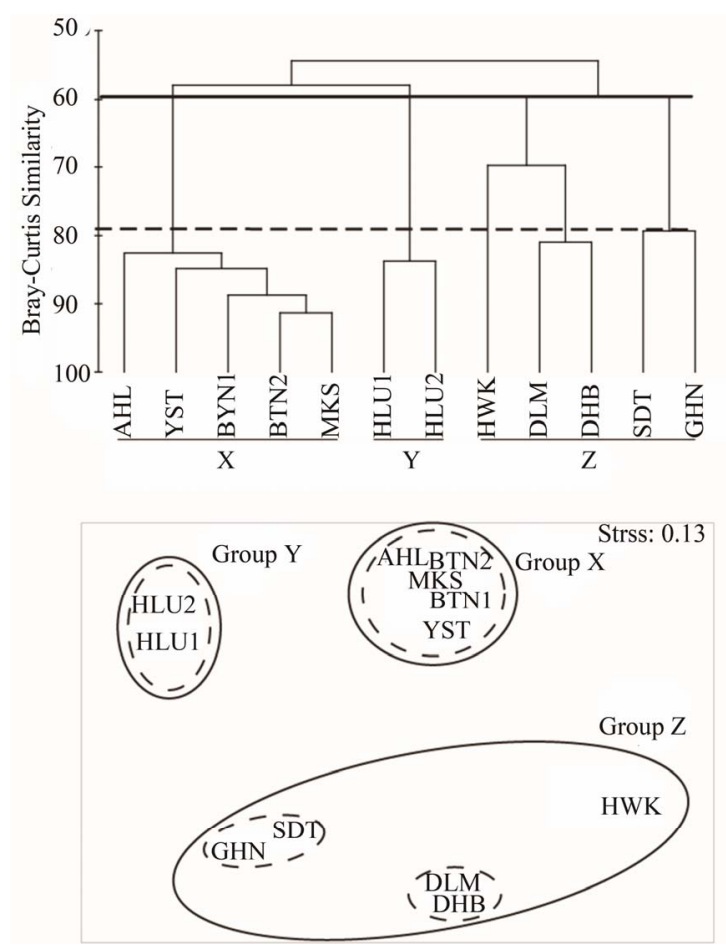

Figure 3. Massive coral assemblages by cluster analysis and non-metric multi-dimensional scaling (nMDS); (upper) Bray-Curtis Similarity cluster analysis depicting three assemblages. (lower) nMDS graphic representation with ovals around assemblages identified by dendrogram. Solid circles indicate groups with $>58 \%$ similarity between sites. Dotted circles indicate subgroups with $>\mathbf{8 0} \%$ similarity. 


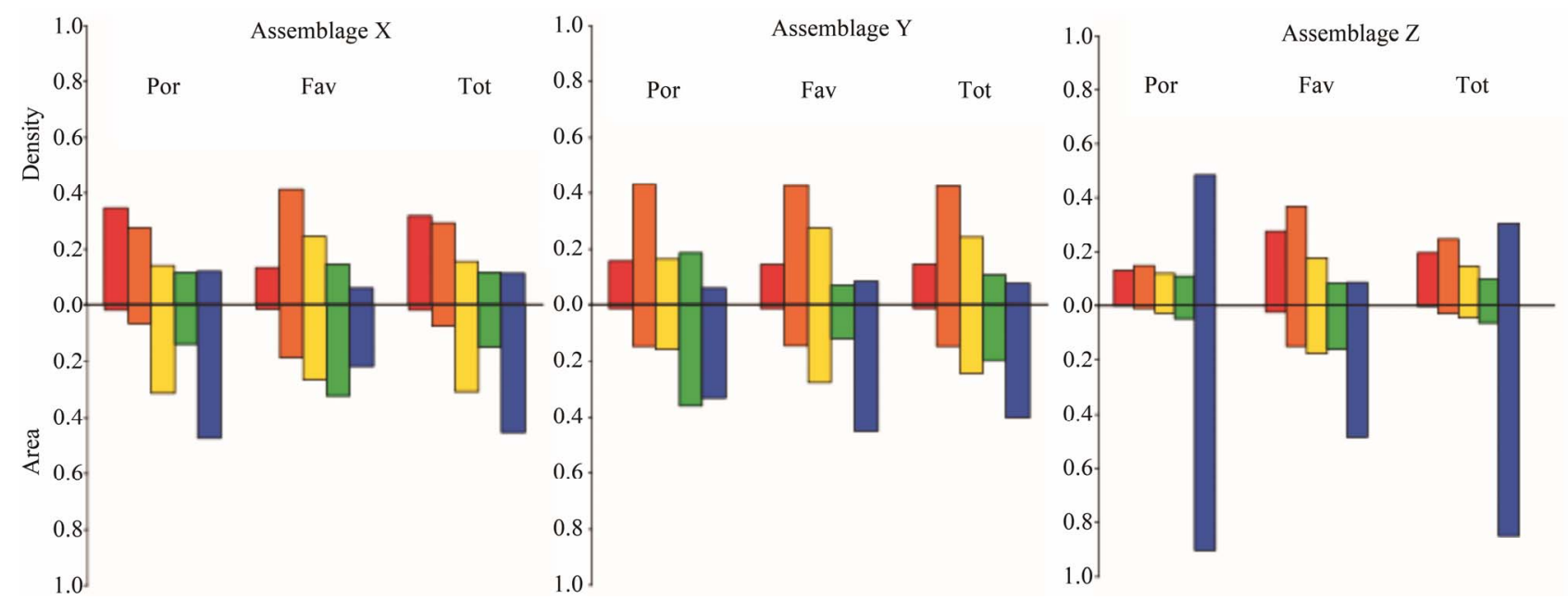

Figure 4. Fractional areas and densities of massive corals; Assemblages determined by cluster analysis. Size classes are shown from smallest (left) to largest (right). POR = Porites; FAV = faviids; Tot = Total massive corals.

Table 3. Biological characteristics of coral assemblages.

\begin{tabular}{|c|c|c|c|c|c|c|c|}
\hline Assemblage Description & Dominant Taxa & Acroporids & $\mathrm{Cov}$ & Den & Gen & $\mathrm{g}_{1}$ & $\mathrm{~g}_{2}$ \\
\hline $\begin{array}{l}\text { X: Sparse Porites harrisoni assemblage } \\
\text { intermingled with other massive species }\end{array}$ & Porites harrisoni & none & $4.6 \pm 1.6$ & $3.9 \pm 1.0$ & 7 & 0.52 & -3.06 \\
\hline $\begin{array}{l}\text { Y: Sparse mixed assemblage of Porites spp., } \\
\text { Acropora spp., faviids and siderastreids }\end{array}$ & None, mixed group & A. clathrata & $3.7 \pm 2.7$ & $5.3 \pm 3.1$ & 9 & 1.28 & 1.11 \\
\hline $\begin{array}{l}\text { Z: Porites harrisoni assemblage, sometimes } \\
\text { with other Porites, massives and acroporids }\end{array}$ & Porites harrisoni & $\begin{array}{l}\text { A. clathrata, } \\
\text { A. arabensis }\end{array}$ & $36.2 \pm 8.3$ & $17.2 \pm 5.2$ & 10 & 0.14 & -1.11 \\
\hline
\end{tabular}

Assemblage descriptions summarize the spatially dominant corals; Dominant taxa are those with the highest density and area cover; Acroporids are those species observed within the assemblage study area; Cov is the percent coral area cover (mean $\pm \mathrm{SE}$ ); Den is the coral density in colonies $\mathrm{m}^{-2}$ (mean $\pm \mathrm{SE}$ ); Gen is the number of coral genera; $g_{1}$ is skewness; $g_{2}$ is kurtosis.

minant massive genera. Subordinate massive genera included Coscinaria, Cyphastrea, Leptastrea and Siderastrea. Acropora comprised $26 \%-55 \%$ of the live coral cover (i.e. $1.0 \%-2.0 \%$ of the total area cover).

Z: This assemblage encompassed three coastal sites near Abu Dhabi (Ras Ghanada, Saadiyat, Dhabiya), one site near Delma Island, and one site situated on top of a limestone dome (Hawksbill Reef). Assemblage Z was moderately populated with Porites harrisoni and other massive species and sparsely populated with two species of Acropora. Porites, Favia/Favites and Platygyra were the predominant massive genera. Subordinate massive genera included Coscinaria, Cyphastrea, Leptastrea, Siderastrea and Turbinaria. Acropora was observed at all sites, except Hawksbill Reef, and comprised $<8 \%$ of the live coral cover (i.e. $<2.2 \%$ of the total area cover). Acanthastrea was observed in Ras Ghanada outside of the transect belts and, therefore, was not included within the assemblage descriptions. Four genera (Plesiastrea, Psammocora, Pseudosiderastrea, and Stylophora spp.) that were inventoried around Dubai before and after the ETAs [7,19] and which have been observed near Ras Ghanada and Saadiyat (Riegl pers. comm.) were not recorded within the monitoring stations.

\subsection{Acropora Community Projections}

Projections for Acropora to attain pre-disturbance levels of $40 \%-90 \%$ area cover [7,8] varied depending on the growth rate, partial mortality and annual recruitment (Figures 5 and 6). In the idealized situations of positive growth only, the current Assemblage $\mathrm{Y}$ and $\mathrm{Z}$ populations will require $15-32$ years to recover from the 2002 ETA and achieve the pre-disturbance area cover of $\geq 40 \%$. This timeframe coincides with other coral regeneration estimates of $10-30$ years (e.g. [27-29]). However, the typical period between mass Acropora mortality events in the Arabian Gulf is $15-17$ years [7,30]. Unless two or more recruits are added to the $\mathrm{Y}$ population and six or more recruits are added to the $\mathrm{Z}$ population each year, regeneration times will exceed the typical disturbance frequency. When growth-limiting factors such as partial mortality are considered, regeneration times will be further delayed.

\section{Discussion}

\subsection{Coral Assemblages in the SE Arabian Gulf}

A census conducted in the spring of 1996 characterized 
(a) Current population, positive and negative growth

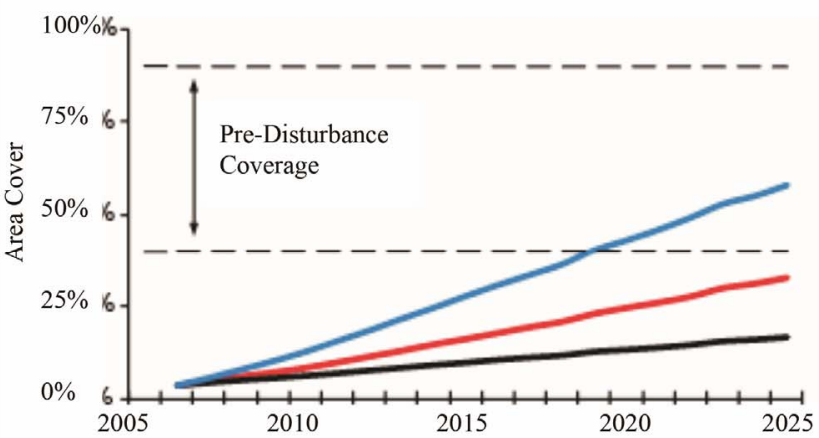

(b) Two recruits per year, positive and negative growth

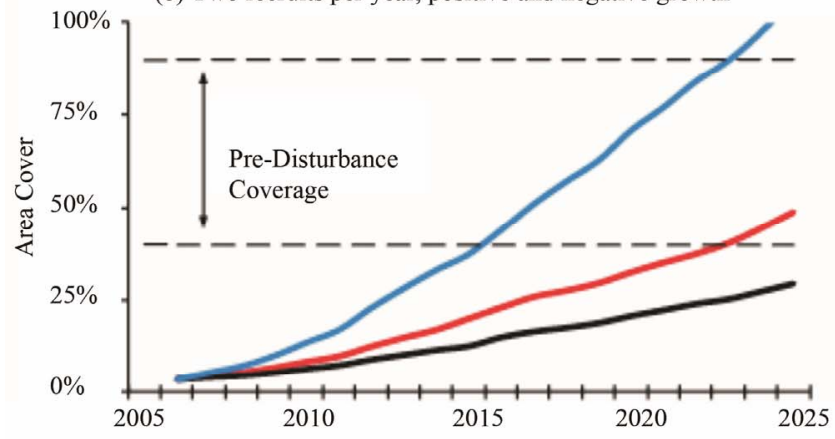

(c) Six recruits per year, positive and negative growth

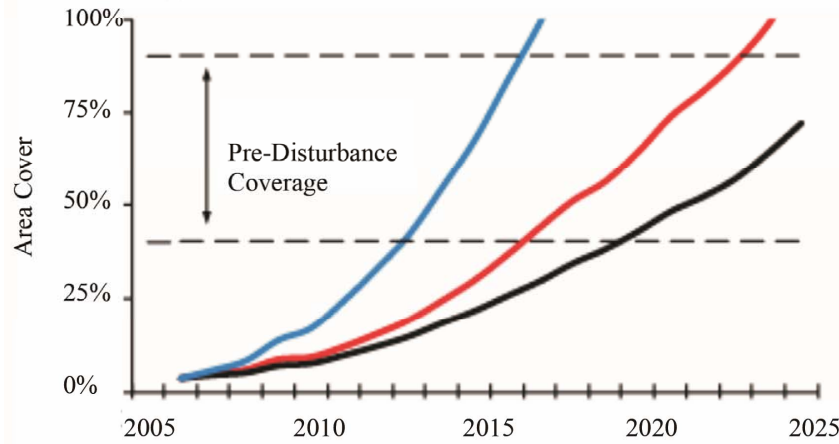

(d) Current population, positive growth only

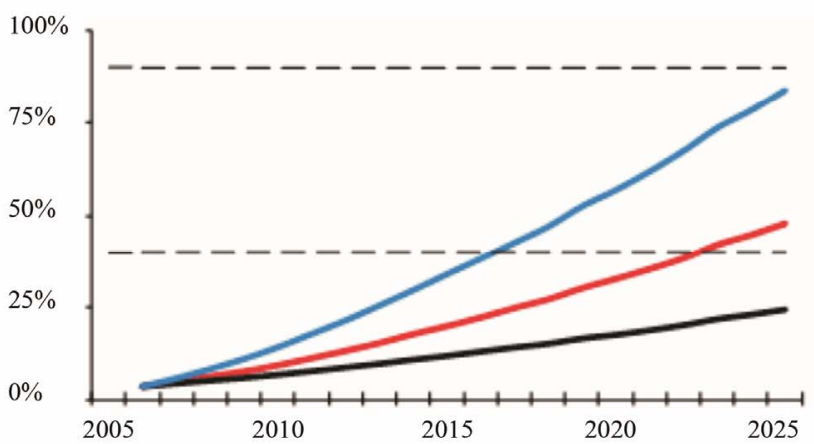

(e) Two recruits per year, positive growth only

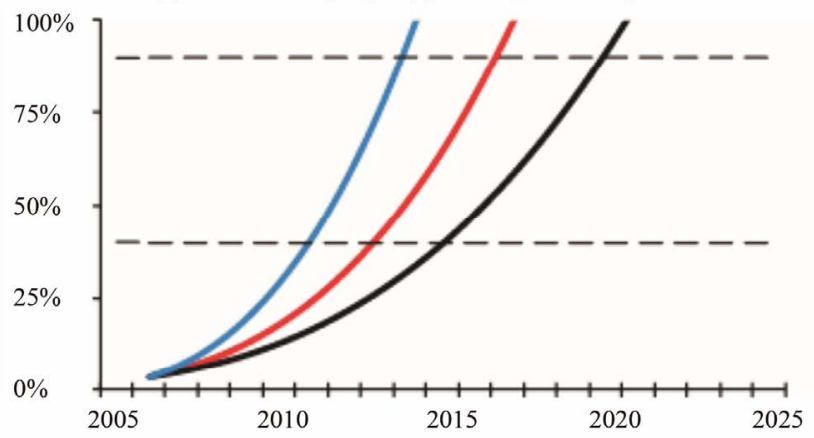

(f) Six recruits per year, positive growth only

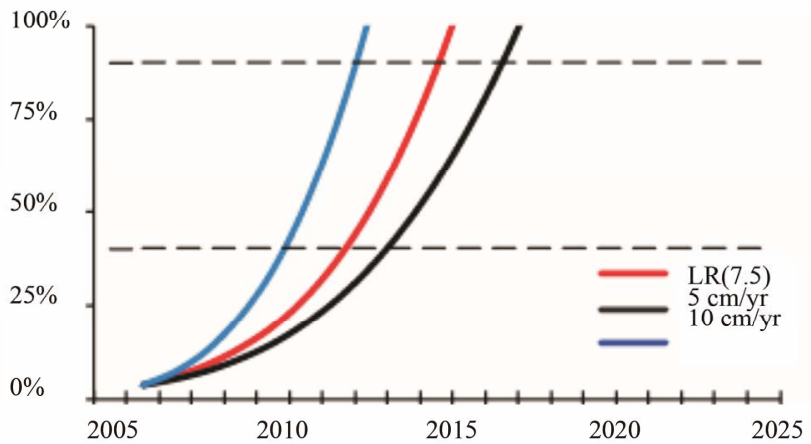

Figure 5. Projections for recovery and colonization of Acropora (Y Assemblage); (a)-(c) Projections include negative growth rates for acroporids similar to those for massive corals in the region (i.e. $40 \%$ of the population undergo a $30 \%$ loss in surface area). (d)-(f) Projections are best case (idealized) situations which do not include any growth-limiting factors.

the southeastern Arabian Gulf coral communities into five well-separated coral assemblages: 1) "A", a Porites lutea assemblage intermingled with other Porites and massive species; 2) "B", a dense Acropora assemblage overtopping subordinate massive corals; 3) "C", a faviid assemblage; 4) "D", a siderastreid assemblage; and 5) "E", a Porites harrisoni assemblage intermingled with other massive species $[7,8,16,20]$. The acroporid populations in Assemblage B suffered mass mortality during the ETA that occurred in the summer of 1996 while the massive coral taxa throughout the region experienced negligible effects during this event and the subsequent anomalies in 1998 and 2002 [7,8]. Post-disturbance studies conducted in 2006-2009 ([19], this study) describe mas- sive coral assemblages that are compositionally similar to the original A, C, and E Assemblages (Figure 7), indicating that the massive taxa groups remained relatively unchanged after the three ETAs. However, the extensive loss of Acropora in 1996 resulted in the fragmentation of Assemblage B into four separate groups in various stages of acroporid recovery; Assemblages X and Y near Abu Dhabi and Qatar, respectively, (this study) and Assemblages 1 and 3 near Dubai [19]. [The fifth of the original assemblages, D, was not observed in the post-disturbance studies.]

Assemblage $\mathrm{X}$ is a sparsely populated Porites harrisoni community intermingled with other massive corals. The presence of consolidated acroporid rubble indicates 

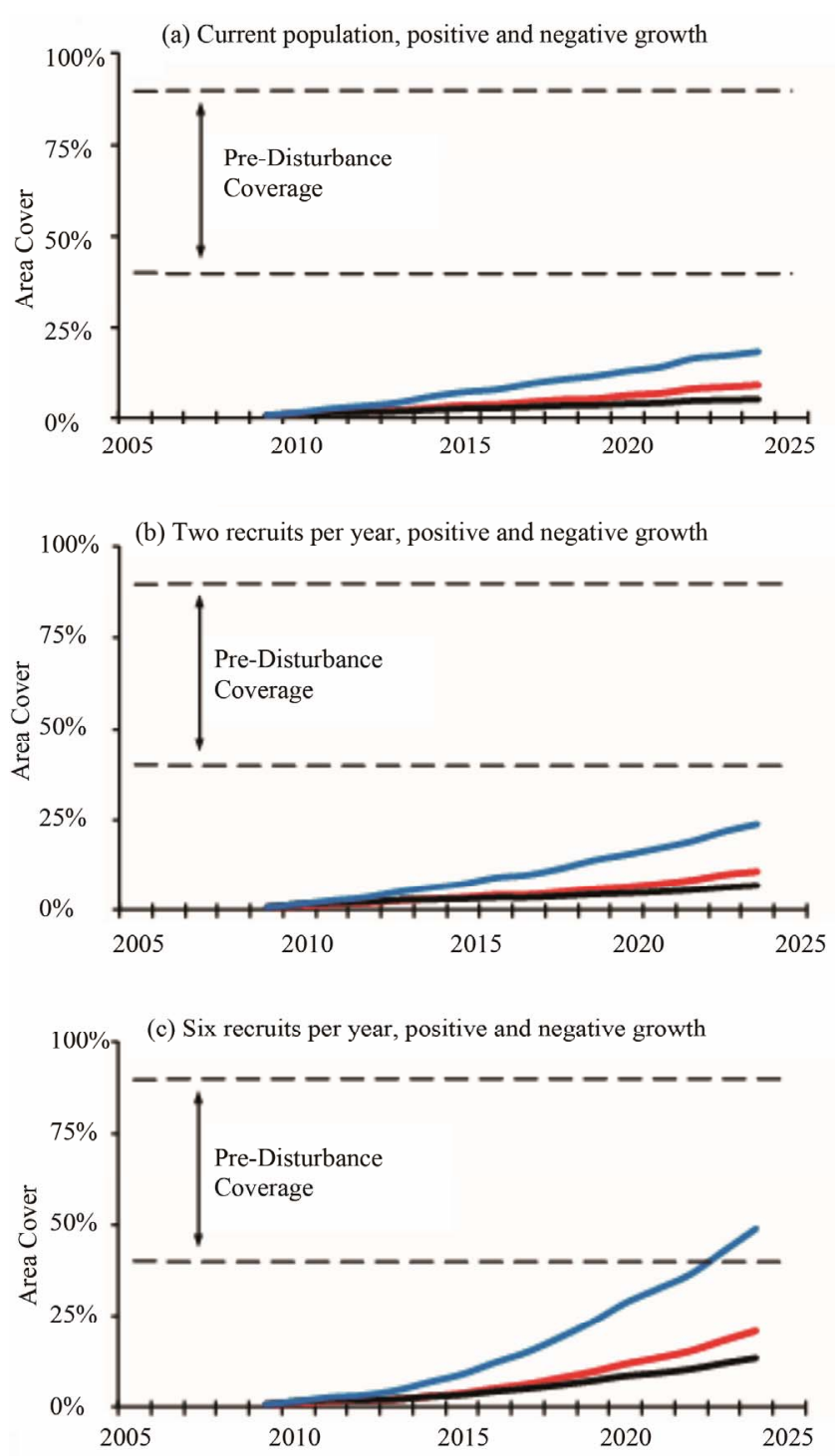

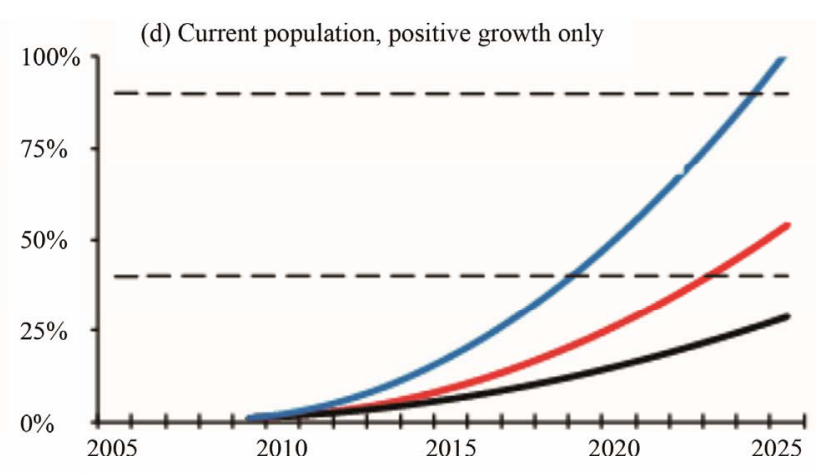

(e) Two recruits per year, positive growth

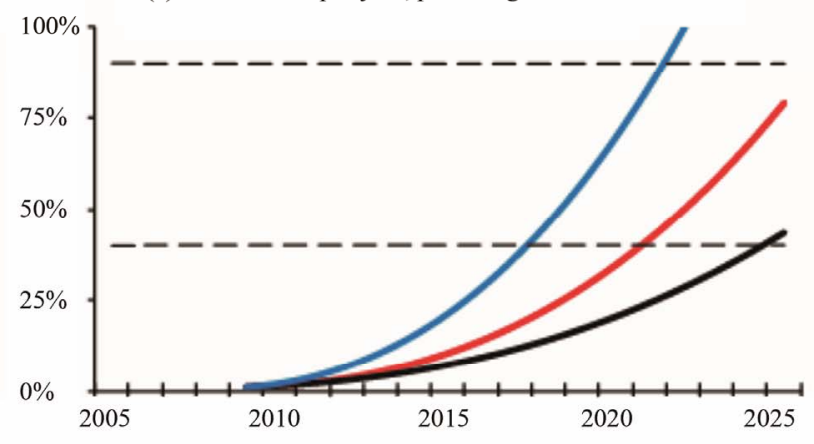

(f) Six recruits per year, positive growth only

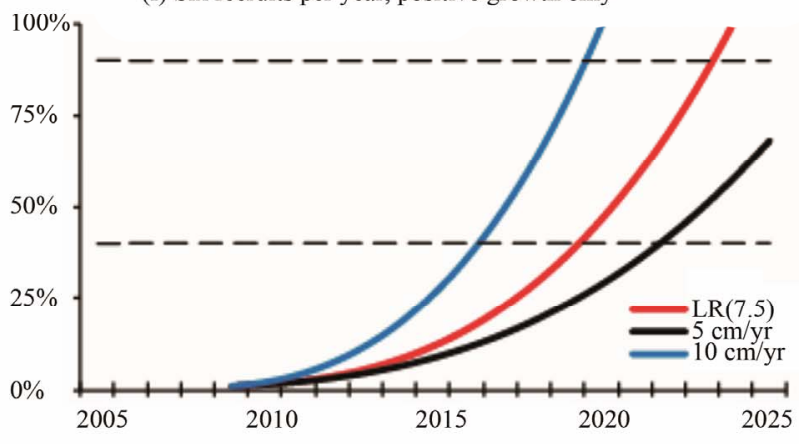

Figure 6. Projections for recovery and colonization of Acropora (Z Assemblage); (a)-(c) Projections include negative growth rates for acroporids similar to those for massive corals in the region (i.e. $40 \%$ of the population undergo a $30 \%$ loss in surface area). (d)-(f) Projections are best case (idealized) situations which do not include any growth-limiting factors.

that these sites were previously suitable habitat for Acropora, although no live acroporids currently exist. Together, the low area cover of massive taxa, the predominance of small colonies (Figure 4), and the prior existence of Acropora indicate that these sites had been compositionally similar to those described as Assemblage B prior to the 1996 disturbance. The absence of acroporids seven years after the third ETA in 2002 suggests a possible local extirpation of acroporids and a phase-shift in coral dominance to poritids and, to a lesser extent, faviids. The Assemblage $X$ sites are located in the southwestern corner of the region (Figure 1), which likely puts them outside the prevailing surface current that can transport competent coral larvae from upstream sources $[31,32]$. Perhaps the multitude of conditions re- quired to reseed this group (e.g. circulation patterns shifted further southwest, an upstream spawning event, and environmental conditions conducive to larval survival and successful settlement) have not happened concurrently since the mass mortality event. The present coral area cover is low $(2 \%-12 \%)$ and is expected to remain low for many years due to the slow growth rates of the massive taxa, leaving substrate available for future acroporid recruits should the conditions become suitable for recolonization.

Assemblage $\mathrm{Y}$ is a sparsely populated mixed community of Porites, Acropora, faviids and other massive corals. The massive coral population resembles that found in Assemblage $X$ and is, therefore, compositionally similar to the understory described as part of Assemblage B prior 


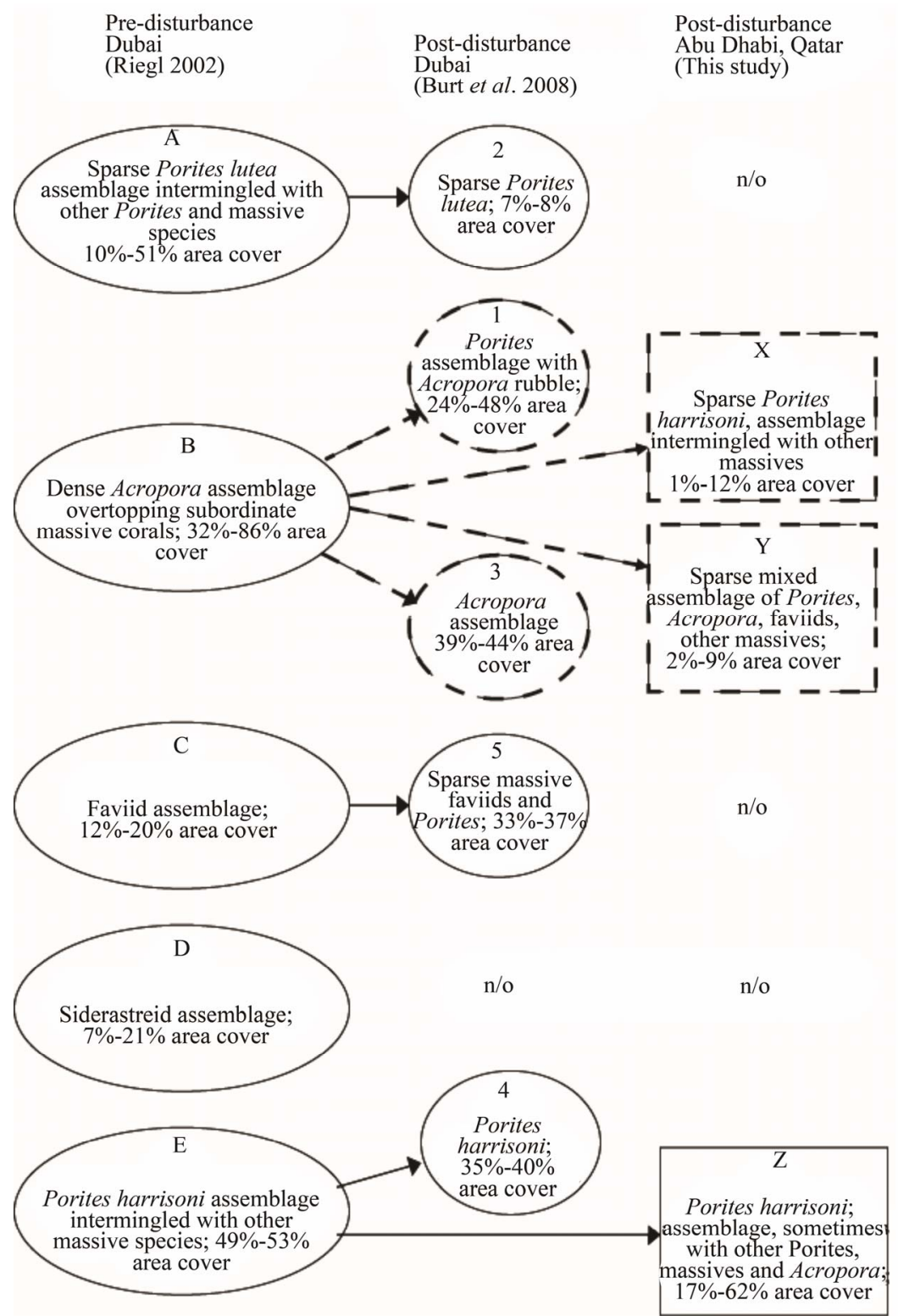

Figure 7. Pre- and post-disturbance coral assemblages; solid arrows link comparable pre- and post-disturbance assemblages. Dotted arrows link post-disturbance assemblages that are in various stages of recovery to the pre-disturbance assemblage. n/o $=$ not observed.

to the 1996 disturbance. The primary difference which separates Assemblages $\mathrm{X}$ and $\mathrm{Y}$ is the presence of eleven Acropora clathrata colonies in the latter group. The Assemblage Y sites are located in the northwestern part of the region (Figure 1), which puts them in the midst of the prevailing surface current that can transport competent coral larvae from Iran, Saudi Arabia or Kuwait [33]. Assemblage $\mathrm{Y}$ is in the early stages of recovery, with acroporids comprising $1 \%-2 \%$ of the total area cover. Projections indicate that recovery of Assemblage $\mathrm{Y}$ acroporids to the $\geq 40 \%$ pre-disturbance area cover will require at least 15 years, provided that the current environmental conditions and coral growth rates remain un- changed. However, when growth-limiting factors such as partial mortality are considered, recovery times may double. Even under idealized conditions, the recovery periods within Assemblage Y meet or exceed the typical 15 - 17 years disturbance cycle in the Arabian Gulf $[7,30]$.

Unlike Assemblages $\mathrm{X}$ and $\mathrm{Y}$, the third group characterized in this study does not resemble a fragmentation of the pre-disturbance Assemblage B. Assemblage Z is a moderately populated community of Porites harrisoni intermingled with other massive species. The massive coral population is compositionally similar to that described as Assemblage E [7,8] prior to the 1996 disturbance. In addition to massive taxa, acroporids have been 
observed at four of the five Assemblage $\mathrm{Z}$ sites (the fifth site, Hawksbill Reef, is a monospecific stand of P. harrisoni) whereas acroporids were not included in the Assemblage $\mathrm{E}$ description. The Assemblage $\mathrm{Z}$ sites are distributed across the southern part of the region with Delma and Hawksbill in the west and Dhabiya, Saadiyat, and Ras Ghanada in the east (Figure 1). The presence of six and sixteen acroporid colonies at Delma and Ras Ghanada, respectively, compared to the single colonies at Dhabiya and Saadiyat and the absence of acroporids at Hawksbill indicates that recruitment within this assemblage does not follow a west-east geographic gradient (i.e. the direction of the prevailing surface current), but may perhaps be influenced by meanders in the current that occur from time to time. Under idealized conditions (i.e. no growth-limiting factors), the current acroporid community could achieve $\geq 40 \%$ area cover after a $17+$ year recovery period (Figure 6(e)) such that Assemblage Z would become compositionally similar to the pre-disturbance Assemblage B (i.e. acroporids become competitively dominant and overtop subordinate massive corals). However, more realistic scenarios that include partial mortality (Figures 6(a)-(c)) indicate that heavy recruitment (i.e. more than six recruits each year) would be necessary for the acroporid area cover to exceed $25 \%$ within the same timeframe; thus acroporids are likely to remain subordinate to $P$. harrisoni and the other massive species within Assemblage Z.

Acroporid recovery in Abu Dhabi and Qatar has differed considerably from that in the adjacent waters of Dubai. Burt et al. (2008) described the recovering acroporid communities in Dubai as follows:

- Assemblage 1-a moderately populated Porites lutea community intermingled with small massive corals, few live Acropora colonies, and extensive acroporid rubble.

- Assemblage 3-a moderately populated Acropora community within which tabular colonies comprised one-third of the $42 \%$ live coral cover.

Juvenile acroporid colonies (15- $20 \mathrm{~cm}$ radii) were observed among both assemblages, indicating post-disturbance recruitment [19]. The presence of large Acropora colonies (50 - $75 \mathrm{~cm}$ radii) among both assemblages [19] indicates that certain individuals survived the 2002 ETA and possibly the preceding event(s). The Dubai acroporids were larger than those found near Abu Dhabi and Qatar which suggests differential survival/growth patterns within the region. Such differences had previously been noted when healthy pockets of Acropora were observed in 1999 both upstream and downstream of the areas near Dubai that had been affected by the mass mortality event [8]. It was speculated that surviving areas, such as the one reported upstream of the damaged Dubai sites near Deira, have helped to repopulate both Dubai assemblages [19]. Assemblage 1 was reported to be in an early stage of recovery, though it is likely further along the recovery trajectory than Assemblage Y near Qatar based on the larger colony sizes within the former group. The broad size distribution and dominance of the acroporids within Assemblage 3 indicated that this group was approaching full recovery faster than all other assemblages described herein.

\subsection{Gradual Re-Emergence of Missing Acropora Species}

Prior to the mass mortality, the acroporid population was comprised of Acropora clathrata and seven other subordinate species (Table 4). After the disturbance, only A. clathrata colonies were reported to have survived as rare individuals among the Assemblage $\mathrm{B}$ sites and also in isolated healthy pockets (e.g. near the Deira Corniche, $75 \mathrm{~km}$ west (downstream) of Abu Dhabi and $30 \mathrm{~km}$ east (upstream) of Dubai) [7,8]; whereas the other Acropora species were completely eliminated.

After a $10+$ year recovery period, three of the "lost" species have been observed in the southeastern Arabian Gulf; A. valenciennesi and A. pharaonis were reported as common and uncommon, respectively, near Dubai [19], and a single colony of A. arabensis has been observed at the Saadiyat site near Abu Dhabi (this study) along with several others in the vicinity between Ras Ghanada and Saadiyat (Riegl pers. comm.). Similar rediscoveries of corals that were presumed lost after mass mortality events have been reported elsewhere (e.g. Millepora boschmai after a 9+ year absence in Panama [34]). The reappearance of three Acropora species indicates that, similar to A. clathrata, these acroporids survived elsewhere in the Gulf in sufficient quantities to reproduce and function as distant larval sources that are reseeding remote locations [32,33]. Such rediscoveries caution against premature conclusions, even a decade after a disturbance, that a species has been permanently extirpated from the region and provide hope that the four Acropora species that remain missing may be found during future surveys.

\subsection{Connectivity Is Key}

Riegl and Purkis (2009) modeled coral community responses to mass mortality events and found that Acropora survival following such disturbances was possible only if one or more stable and sufficiently sizeable populations survived to become larval donors for the impacted populations. The results of this study provide a real-world example that supports their model. Because the Acropora populations near Qatar and Abu Dhabi are too small in size and number to be self-seeding, these sites are dependent upon larval supplies from distant 
Table 4. Acropora species inventoried pre- and post-disturbance.

\begin{tabular}{cccc}
\hline $\begin{array}{c}\text { Pre-Disturbance (1996) } \\
\text { Dubai }\end{array}$ & $\begin{array}{c}\text { Post-Disturbance (1996) } \\
\text { Dubai }\end{array}$ & Post-Disturbance (2006) ${ }^{*}$ Dubai & $\begin{array}{c}\text { Post-Disturbance (2006-2009) } \\
\text { Abu Dhabi, Qatar }\end{array}$ \\
\hline A. clathrata & A. clathrata & A. clathrata & A. clathrata \\
A. valenciennesi & -- & A. valenciennesi & -- \\
A. pharaonis & -- & A. pharaonis (uncommon) & -- \\
A. arabensis & -- & -- & A. arabensis (rare) \\
A. horrida & -- & -- & -- \\
A. florida & -- & -- & -- \\
A. valida & -- & -- & -- \\
A. tenuis & -- & -- & -
\end{tabular}

Sources: ${ }^{*}$ Riegl (1999), ${ }^{+}$Burt et al. (2008), ${ }^{* *}$ This study.

upstream refuges. This connectivity reinforces the necessity for multi-national cooperation to protect coral species. In the Arabian Gulf, stressors that affect Acropora in Iran, Saudi Arabia, and Kuwait [11] can have a direct impact on the ability of coral communities in Qatar and the United Arab Emirates to recover following a mass mortality event.

Projections for Acropora recovery and colonization in the $\mathrm{Y}$ and $\mathrm{Z}$ Assemblages demonstrate the importance of continuous annual recruitment (Figures 5 and 6). The acroporid community near Halul, Qatar (Assemblage Y) is growing and may soon become large enough to self-seed: nine of the eleven colonies have diameters greater than $6.25 \mathrm{~cm}$, the size beyond which Acropora are generally reproductive [35]. Among the five $\mathrm{Z}$ sites, only Delma and Ras Ghanada had multiple Acropora colonies; however, each had just two individuals that were large enough to be reproductive. (The remaining juveniles could reach the typical size for sexual maturity within 1 - 2 years and may then become capable of self-seeding.) Until each local community can develop its own self-seeding population, recruitment from connected larval sources shall remain critical.

Acroporid recovery and colonization projections indicate that a disturbance-free period of 15 - 32 years is required to attain pre-1996 Acropora abundance levels. However, mass Acropora mortality events have been occurring at approximately 15 - 17 year cycles in the Arabian Gulf [7,30] and may occur more frequently as a result of climate change [17]. It is possible, and perhaps likely, that acroporids shall experience continual changes in population structure due to persistent cycles of regeneration and mass mortality and that larval refuges shall play an increasingly important role in re-seeding impacted sites. It is recommended that multi-national efforts be put forth to identify these refuges, establish the connectivity between coral communities, and institute appropriate management and conservation programs.

\section{Acknowledgements}

This research began as a three-year project to study the coral reefs of Abu Dhabi and Eastern Qatar, sponsored by Dolphin Energy and managed by the Emirates Wildlife Society in association with World Wide Fund for Nature (EWS-WWF). Post-project work continued through the support of the National Coral Reef Institute (NCRI) and the Environment Agency-Abu Dhabi (EAD). Special thanks go to Dr. Bernhard Riegl (NCRI), Ashraf Al-Cibahy (EAD), and Thabit Al-Abdessalaam (EAD) for their continuous support throughout the entirety of this study. Additional thanks go to Ibrahim Bugla (EAD) for all of the field work and logistics coordination in Abu Dhabi; Dr. Mohamed Alaa Abdel-Moati of the Supreme Council for the Environment and Natural Reserves (SCENR) for management and logistics coordination in Qatar; and Dr. Sam Purkis (NCRI), Dr. Fred Launay (EWS-WWF) and Razan Al Mubarak (EWS-WWF) for their efforts from the inception through the end of the original project. Many other EAD and SCENR staff, rangers and boat drivers contributed during the field work over many years-Shukran to all.

\section{REFERENCES}

[1] C. Wilkinson, "Status of Coral Reefs of the World: 1998," Australian Institute of Marine Science, Townsville, 1998.

[2] T. Goreau, T. McClanahan, R. Hayes and A. Strong, "Conservation of Coral Reefs after the 1998 Global Bleaching Event," Conservation Biology, Vol. 14, No. 1, 2000, pp. 5-15. doi:10.1046/j.1523-1739.2000.00011.x

[3] C. Wilkinson, "Status of the Coral Reefs of the World: 2000," Australian Institute of Marine Science, Townsville, 2000 . 
[4] J. F. Bruno, C. Siddon, J. D. Whitman, P. L. Colin and M. A. Toscano, "El Niño Related Coral Bleaching in Palau, Western Caroline Islands," Coral Reefs, Vol. 20, No. 2, 2001, pp. 127-136. doi:10.1007/s003380100151

[5] P. J. Mumby, J. R. M. Chisholm, A. J. Edwards, C. D. Clark, E. B. Roark, S. Andrefouet and J. Jaubert, "Unprecedented Bleaching-Induced Mortality in Porites spp. at Rangiron Atoll, French Polynesia," Marine Biology, Vol. 139, No. 1, 2001, pp. 183-189.

doi:10.1007/s002270100575

[6] R. B. Aronson, W. F. Precht, M. A. Toscano and K. H. Koltes, "The 1998 Bleaching Event and its Aftermath on a Coral Reef in Belize," Marine Biology, Vol. 141, No. 3, 2002, pp. 435-447. doi:10.1007/s00227-002-0842-5

[7] B. Riegl, "Coral in a Non-Reef Setting in the Southern Arabian Gulf (Dubai, UAE): Fauna and Community Structure in Response to Recurring Mass Mortality," Coral Reefs, Vol. 18, No. 1, 1999, pp. 63-73. doi: $10.1007 / \mathrm{s} 003380050156$

[8] B. Riegl, "Effects of the 1996 and 1998 Positive SeaSurface Temperature Anomalies on Corals, Coral Diseases and Fish in the Arabian Gulf (Dubai, UAE)," Marine Biology, Vol. 140, No. 1, 2002, pp. 29-40. doi: $10.1007 / \mathrm{s} 002270100676$

[9] D. J. J. Kinsman, "Reef Coral Tolerance of High Temperatures and Salinities," Nature, Vol. 202, No. 4939, 1964, pp. 1280-1282. doi:10.1038/2021280a0

[10] E. A. Shinn, "Coral Reef Recovery in Florida and the Persian Gulf," Environmental Geology, Vol. 1, No. 4, 1976, pp. 241-254. doi:10.1007/BF02407510

[11] K. E. Carpenter, E. P. L. Harrison, G. Hodgson, A. H. Asaffar and S. H. Alhazeem, "The Corals and Coral Reef Fishes of Kuwait," Kuwait Institute for Scientific Research, Kuwait, 1997.

[12] S. L. Coles, "Coral Species Diversity and Environmental Factors in the Arabian Gulf and the Gulf of Oman: A Comparison to the Indo-Pacific Region," Atoll Research Bulletin, Vol. 507, 2003, pp. 497-508. doi:10.5479/si.00775630.507.1

[13] H. Rezai, S. C. Wilson, M. Claereboudt and B. Riegl, "Coral Reef Status in the ROPME Sea Area: Arabian/Persian Gulf, Gulf of Oman and Arabian Sea," In: C. Wilkinson, Ed., Status of Coral Reefs of the World, 2004, pp. $155-170$.

[14] M. R. Claereboudt, "Reef Corals and Coral Reefs of the Gulf of Oman," Historical Association of Oman, Oman, 2006.

[15] C. R. C. Sheppard and R. Loughland, "Coral Mortality and Recovery in Response to Increasing Temperature in the Southern Arabian Gulf," Aquatic Ecosystem Health, Vol. 5, No. 4, 2002, pp. 395-402. doi:10.1080/14634980290002020

[16] B. Riegl, "Climate Change and Coral Reefs: Different Effects in Two High-Latitude Areas (Arabian Gulf, South Africa)," Coral Reefs, Vol. 22, No. 4, 2003, pp. 433-446. doi:10.1007/s00338-003-0335-0

[17] C. R. C. Sheppard, "Predicted Recurrences of Mass Coral Mortality in the Indian Ocean," Nature, Vol. 425, No.
6955, 2003, pp. 294-297. doi:10.1038/nature01987

[18] S. L. Coles and B. E. Brown, "Coral Bleaching-Capacity for Acclimatization and Adaptation," Advances in Marine Biology, Vol. 46, 2003, pp. 183-223. doi:10.1016/S0065-2881(03)46004-5

[19] J. Burt, A. Bartholomew and P. Usseglio, "Recovery of Corals a Decade after a Bleaching Event in Dubai, United Arab Emirates," Marine Biology, Vol. 154, No. 1, 2008, pp. 27-36. doi:10.1007/s00227-007-0892-9

[20] B. Riegl, "Inhibition of Reef Framework by Frequent Disturbance: Examples from the Arabian Gulf, South Africa, and the Cayman Islands," Palaeogeography, $\mathrm{Pa}$ laeoclimatology, Palaeoecology, Vol. 175, No. 1-4, 2001, pp. 79-101. doi:10.1016/S0031-0182(01)00387-X

[21] K. E. Kohler and S. M. Gill, "Coral Point Count with Excel Extensions (CPCe): A Visual Basic Program for the Determination of Coral and Substrate Coverage using Random Point Count Methodology," Computers \& Geosciences, Vol. 32, No. 9, 2006, pp. 1259-1269. doi:10.1016/j.cageo.2005.11.009

[22] R. M. Reynolds, "Physical Oceanography of the Gulf, Strait of Hormuz, and the Gulf of Oman-Results from the Mt Mitchell Expedition," Marine Pollution Bulletin, Vol. 27, 1993, pp. 35-59. doi:10.1016/0025-326X(93)90007-7

[23] K. R. Clarke and R. N. Gorley, "PRIMER v6: User Manual/Tutorial," PRIMER-E, Plymouth, 2006.

[24] B. M. Riegl, S. J. Purkis, J. Keck and G. P. Rowlands, "Monitored and Modeled Coral Population Dynamics and the Refuge Concept," Marine Pollution Bulletin, Vol. 58, No. 1, 2009, pp. 24-38. doi:10.1016/j.marpolbul.2008.10.019

[25] H. Caswell, "Matrix Population Models: Construction, Analysis, and Interpretation," Sinauer Associates, Inc., Sunderland, 2001.

[26] N. Owen-Smith, "Introduction to Modeling in Wildlife and Resource Conservation," Blackwell Publishing, Malden, 2007.

[27] J. H. Connell, "Disturbance and Recovery of Coral Assemblages," Coral Reefs, Vol. 16, No. 5, 1997, pp. S101S113. doi: $10.1007 / \mathrm{s} 003380050246$

[28] R. Niñio, M. Meekan, T. Done and H. Sweatman, "Temporal Patterns in Coral Assemblages on the Great Barrier Reef from Local to Large Spatial Scales,' Marine Ecology Progress Series, Vol. 194, 2000, pp. 65-74. doi:10.3354/meps 194065

[29] J. F. Bruno and E. R. Selig, "Regional Decline of Coral Cover in the Indo-Pacific: Timing, Extent and Subregional Comparisons," PLoS One, Vol. 2, No. 8, 2007, Article ID: e711. doi:10.1371/journal.pone.0000711

[30] B. M. Riegl and S. J. Purkis, "Model of Coral Population Response to Accelerated Bleaching and Mass Mortality in a Changing Climate," Ecological Modelling, Vol. 220, No. 2, 2009, pp. 192-208.

doi:10.1016/j.ecolmodel.2008.09.022

[31] P. L. Harrison and C. C. Wallace, "Reproduction, Dispersal and Recruitment of Scleractinian Corals," In: Z. Dubinsky, Ed., Coral Reef Ecosystems, Ecosystems of the World, Elsevier Science Publishers, Amsterdam, 1990, pp. 
133-207.

[32] P. L. Harrison, "Sexual Reproduction of Scleractinian Corals," Coral Reefs: An Ecosystem in Transition, Part 3, Springer Publishers, 2011, pp. 59-85.

doi:10.1007/978-94-007-0114-4_6

[33] P. L. Harrison, "Status of the Coral Reefs of Kuwait," Final Report to the United Nations Industrial Development Organization and the United Nations Development
Programme, 1995.

[34] P. W. Glynn and J. S. Feingold, "Hydrocoral Species Not Extinct," Science, Vol. 257, No. 5078, 1992, p. 1845.

[35] V. R. Hall and T. P. Hughes, "Reproductive Strategies of Modular Organisms: Comparative Studies of Reef-Building Corals," Ecology, Vol. 77, No. 3, 1996, pp. 950-963. doi:10.2307/2265514 